\title{
ON MAXIMA OF TAKAGI-VAN DER WAERDEN FUNCTIONS
}

\author{
YOSHIKAZU BABA
}

\begin{abstract}
Generalizing Takagi's function $F_{2}(x)$ and van der Waerden's function $F_{10}(x)$, we introduce a class of nowhere differentiable continuous functions $F_{r}(x), r \geq 2$. Some properties of $F_{r}(x)$ concerning especially maxima are discussed. When $r$ is even, the Hausdorff dimension of the set of $x$ 's giving the maxima of $F_{r}(x)$ is proved to be $1 / 2$.
\end{abstract}

1. Introduction. Let $d(x)$ be the distance from $x$ to the nearest integer. The function $d(x)$ is continuous and periodic with period 1. Fix an integer $r \geq 2$ and define $F_{r}^{n}(x)=\sum_{k=0}^{n} d\left(r^{k} x\right) / r^{k}$. When $n$ tends to infinity, $F_{r}^{n}(x)$ converges uniformly to a continuous and periodic (with period 1) function $F_{r}(x)$. Further, $F_{r}(x)$ is proved to be everywhere nondifferentiable. As a simple example of a nowhere differentiable continuous function, T. Takagi $[\mathbf{1}]$ discovered $F_{2}(x)$ and a quarter of a century later B. L. van der Waerden [2] rediscovered $F_{10}(x)$. Takagi's proof of the nowhere differentiability of $F_{2}(x)$ is applicable to any $r \geq 3$ with a slight modification when $r$ is odd. Recently, B. Martynov [3] discussed the structure of the set $E_{2}=\left\{0 \leq x \leq 1 ; F_{2}(x)=M_{2}\right\}$ where $M_{2}=\max F_{2}(x)$ and the result is that $x=0 . x_{1} x_{2} \cdots x_{n} \cdots$ (the base-4 expansion of $x$ ) belongs to $E_{2}$ if and only if $x_{n}=1$ or 2 for any $n \geq 1$. From this result we can easily see that the Hausdorff dimension of $E_{2}$ is equal to $\log 2 / \log 4=1 / 2$. This has a relation to the fact that the Hausdorff dimension of the set of zeros of the Brownian motion $B(t, \omega)$ is equal to $1 / 2$ and the sample functions of $B(t, \omega)$ are nowhere differentiable continuous ones for almost all $\omega$. In this paper we show that for any even $r \geq 2$ the Hausdorff dimension of the set $E_{r}=\left\{0 \leq x \leq 1 ; F_{r}(x)=M_{r} \equiv \max F_{r}(x)\right\}$ is equal to $1 / 2$ generalizing Martynov's arguments to $r \geq 2$.

\section{Functional equations.}

PROPOSITION 1. The function $F_{r}(x)$ satisfies the following functional equations:

$$
\begin{gathered}
F_{r}(r x)=r F_{r}(x)-r d(x), \\
F_{r}(x)=F_{r}^{1}(x)+\frac{1}{r^{2}} F_{r}\left(r^{2} x\right) .
\end{gathered}
$$

ProOF. First,

$$
\begin{aligned}
F_{r}^{n}(r x) & =r\left(d(r x) / r+\cdots+d\left(r^{n+1} x\right) / r^{n+1}\right) \\
& =r\left(F_{r}^{n+1}(x)-d(x)\right) .
\end{aligned}
$$

Received by the editors September 9, 1983.

1980 Mathematics Subject Classification. Primary 26A27; Secondary 60G17.

Key words and phrases. Nowhere differentiable continuous function, Hausdorff dimension. 
Taking the limit of the both sides, we have (1). Next,

$$
\begin{aligned}
F_{r}\left(r^{2} x\right) & =r F_{r}(r x)-r d(r x)=r\left(r F_{r}(x)-r d(x)\right)-r d(r x) \\
& =r^{2}\left(F_{r}(x)-F_{r}^{1}(x)\right) .
\end{aligned}
$$

This implies (2).

PROPOSITION 2. The function $F_{r}(x)$ is the unique bounded solution of the functional equation

$$
f(r x)=r f(x)-r d(x) \text {. }
$$

PROOF. Substituting $r^{k-1} x$ for $x$ in $\left(1^{\prime}\right)$ and dividing both sides of the resulting equation by $r^{k}$, we have

$$
\frac{f\left(r^{k} x\right)}{r^{k}}=\frac{f\left(r^{k-1} x\right)}{r^{k-1}}-\frac{d\left(r^{k-1} x\right)}{r^{k-1}}
$$

Summing up these for $k=1$ to $n$, we have

$$
\frac{f\left(r^{n} x\right)}{r^{n}}=f(x)-\sum_{k=0}^{n-1} \frac{d\left(r^{k} x\right)}{r^{k}}
$$

Letting $n \rightarrow \infty$ in the both sides, we obtain $f(x)=F_{r}(x)$.

REMARK. The functional equation $\left(1^{\prime}\right)$ for $r=2$ is a special case of the functional equation studied by M. Yamaguti and M. Hata [4].

3. $E_{r}$ and $M_{r}$. Observing the graphs of the functions $F_{r}^{\mathbf{1}}(x), F_{r}^{2}(x), \ldots$, we can easily see that (i) if $r$ is odd, then $E_{r}=\{1 / 2\}$ and

$$
M_{r}=F_{r}\left(\frac{1}{2}\right)=\frac{1}{2}\left(1+\frac{1}{r}+\frac{1}{r^{2}}+\cdots\right)=\frac{r}{2 r-2}
$$

and (ii) if $r$ is even, then $F_{r}^{1}(x)=1 / 2$ for all $x$ in the interval $[(r-1) / 2 r,(r+1) / 2 r]$. Therefore, in the following, we consider only the case of even $r$ 's.

PROPOSITION 3 .

$$
\begin{gathered}
E_{r} \subset\left[\frac{r}{2 r+2}, \frac{r+2}{2 r+2}\right] \quad\left(\subset\left[\frac{r-1}{2 r}, \frac{r+1}{2 r}\right]\right), \\
M_{r}=\frac{r^{2}}{2 r^{2}-2} \quad\left(=F_{r}\left(\frac{r}{2 r+2}\right)=F_{r}\left(\frac{r+2}{2 r+2}\right)\right) .
\end{gathered}
$$

PROOF. First, by the periodicity of the function $F_{r}(x)$, we have

$$
\begin{aligned}
F_{r}\left(\frac{r}{2 r+2}\right) & =F_{r}^{1}\left(\frac{r}{2 r+2}\right)+\frac{1}{r^{2}} F_{r}\left(\frac{r^{3}}{2 r+2}\right) \\
& =\frac{1}{2}+\frac{1}{r^{2}} F_{r}\left(\frac{r}{2 r+2}\right) .
\end{aligned}
$$

Therefore $F_{r}(r /(2 r+2))=r^{2} /\left(2 r^{2}-2\right)$ and this is equal to $F_{r}((r+2) /(2 r+2))$ since the function $F_{r}(x)$ is symmetric with respect to $1 / 2$. Next, take $x \in E_{r}$ with $x \leq 1 / 2$. Then $F_{r}(r x)=r F_{r}(x)-r d(x)=r F_{r}(x)-r x \leq M_{r}$, therefore we have

$$
x \geq \frac{r-1}{r} M_{r} \geq \frac{r-1}{r} F_{r}\left(\frac{r}{2 r+2}\right)=\frac{r}{2 r+2} .
$$


From this and by the symmetric property of $F_{r}(x)$ follows (3). To obtain (4) take $x \in E_{r}$, then

$$
M_{r}=F_{r}(x)=F_{r}^{1}(x)+\frac{1}{r^{2}} F_{r}\left(r^{2} x\right) \leq \frac{1}{2}+\frac{1}{r^{2}} M_{r}
$$

From this follows

$$
M_{r} \leq \frac{1}{2\left(1-1 / r^{2}\right)}=\frac{r^{2}}{2 r^{2}-2}=F_{r}\left(\frac{r}{2 r+2}\right),
$$

obtaining (4).

Proposition 4. (i) If $x \in E_{r}$, then the fractional part of $r^{2} x\left(\equiv\left\{r^{2} x\right\}\right)$ also belongs to $E_{r}$.

(ii) If $F_{r}\left(r^{2} x\right)=M_{r}$ and $x \in[(r-1) / 2 r,(r+1) / 2 r]$, then $x \in E_{r}$.

PROOF. (i) If $x \in E_{r}$, then

$$
\frac{r^{2}}{2\left(r^{2}-1\right)}=F_{r}(x)=\frac{1}{2}+\frac{1}{r^{2}} F_{r}\left(r^{2} x\right) .
$$

Therefore $F_{r}\left(r^{2} x\right)=r^{2} / 2\left(r^{2}-1\right)$ from which follows $\left\{r^{2} x\right\} \in E_{r}$ by the periodicity of $F_{r}(x)$.

(ii) By the assumptions we have

$$
F_{r}(x)=\frac{1}{2}+\frac{1}{r^{2}} F_{r}\left(r^{2} x\right)=\frac{1}{2}+\frac{1}{2\left(r^{2}-1\right)}=\frac{r^{2}}{2\left(r^{2}-1\right)}
$$

obtaining $x \in E_{r}$.

Proposition 5. Suppose $x \in E_{r}$ and $k$ is a positive integer. Then we have $(x+k) / r^{2} \in E_{r}$ if and only if $\left(r^{2}-r\right) / 2 \leq k \leq\left(r^{2}+r-2\right) / 2$.

PROOF. If $(x+k) / r^{2} \in E_{r}$, then

$$
\frac{r}{2 r+2} \leq \frac{x+k}{r^{2}} \leq \frac{r+2}{2 r+2}
$$

To satisfy these inequalities, it is necessary for $k$ to be in the interval $\left[\left(r^{2}-r\right) / 2\right.$, $\left.\left(r^{2}+r-2\right) / 2\right]$ as is easily checked. Conversely, if $\left(r^{2}-r\right) / 2 \leq k \leq\left(r^{2}+r-2\right) / 2$, then for $x \in E_{r}$ the inequalities

$$
\frac{r-1}{2 r} \leq \frac{x+k}{r^{2}} \leq \frac{r+1}{2 r}
$$

hold as is easily seen and $F_{r}\left(r^{2}(x+k) / r^{2}\right)=F_{r}(x+k)=M_{r}$ because of the periodicity of $F_{r}(x)$. By (ii) of Proposition 4 follows $(x+k) / r^{2} \in E_{r}$.

REMARK. The number of the integers $k$ satisfying the inequalities $\left(r^{2}-r\right) / 2 \leq$ $k \leq\left(r^{2}+r-2\right) / 2$ is equal to $r$.

4. Base- $r^{2}$ expansion. Put $\alpha=\left(r^{2}-r\right) / 2$ and $\beta=\left(r^{2}+r-2\right) / 2$. Let us expand the smallest and the largest numbers of $E_{r}$ into base- $r^{2}$ decimals $\left(r^{2}\right.$-mals). Then

and

$$
\frac{r}{2 r+2}=\left(\sum_{n=1}^{\infty} \frac{1}{\left(r^{2}\right)^{n}}\right) \alpha=0 . \alpha \alpha \cdots \alpha \cdots=0 . \dot{\alpha}
$$

$$
\frac{r+2}{2 r+2}=\left(\sum_{n=1}^{\infty} \frac{1}{\left(r^{2}\right)^{n}}\right) \beta=0 . \beta \beta \cdots \beta \cdots=0 . \dot{\beta} .
$$


Generally we have the following proposition.

PROPOSITION 6. Let $x=0 . x_{1} x_{2} \cdots x_{n} \cdots$ be the base- $r^{2}$ expansion of $x \in[0,1]$. Then, $x \in E_{r}$ if and only if $\alpha \leq x_{n} \leq \beta$ for any $n \geq 1$.

PROOF. Since $r /(2 r+2)$ and $(r+2) /(2 r+2)$ belong to $E_{r}$, if integers $x_{1}, x_{2}, \ldots$, $x_{n}$ are contained in the interval $[\alpha, \beta]$, then by applying $n$ times (ii) of Proposition 4 , we have $0 . x_{1} x_{2} \cdots x_{n} \dot{\alpha} \in E_{r}$ and $0 . x_{1} x_{2} \cdots x_{n} \dot{\beta} \in E_{r}$. From this follows $0 . x_{1} x_{2} \cdots$ $x_{n} \cdots \in E_{r}$ by the continuity of $F_{r}(x)$. Conversely, if there exists an integer $x_{n} \notin$ $[\alpha, \beta]$ in the base- $r^{2}$ expansion $0 . x_{1} x_{2} \cdots x_{n} \cdots \in E_{r}$, then by using $n-1$ times (i) of Proposition 4, we have $0 . x_{n} x_{n+1} \cdots \in E_{r}$ and this contradicts to $E_{r} \subset[0 . \dot{\alpha}, 0 . \dot{\beta}]$.

\section{Hausdorff dimension.}

THEOREM. For any even integer $r \geq 2$, we have $\operatorname{dim} E_{r}=1 / 2$.

PROOF. For all positive integers $n, E_{r}$ can be covered by $r^{n}$, and no fewer than $r^{n}$, intervals of length $r^{-2} n$. Therefore, for every positive real number $s<1, E_{r}$ can be covered by $r^{n+1}$, and no fewer than $r^{n}$, intervals of length $s$, where $n=$ $[-\log s / 2 \log r]$. But

$$
\lim _{s \rightarrow 0} \log r^{n} /(-\log s)=\lim _{s \rightarrow 0} \log r^{n+1} /(-\log s)=1 / 2,
$$

so $\operatorname{dim} E_{r}=1 / 2$.

ACKNOWLEDGEMENT. I am grateful to the referee for simplifying the proof of the theorem.

\section{REFERENCES}

1. T. Takagi, A simple example of the continuous function without derivative, Proc. Phys.-Math. Soc. Tokyo Ser. II 1 (1903), 176-177.

2. B. L. van der Waerden, Ein einfaches Beispiel einer nichtdifferenzierbaren stetigen Funktion, Math. Z. 32 (1930), 474-475.

3. B. Martynov, On maxima of the van der Waerden function, Kvant, June 1982, 8-14. (Russian) 4. M. Yamaguti and M. Hata, Weierstrass's function and chaos, Hokkaido Math. J. (to appear). 